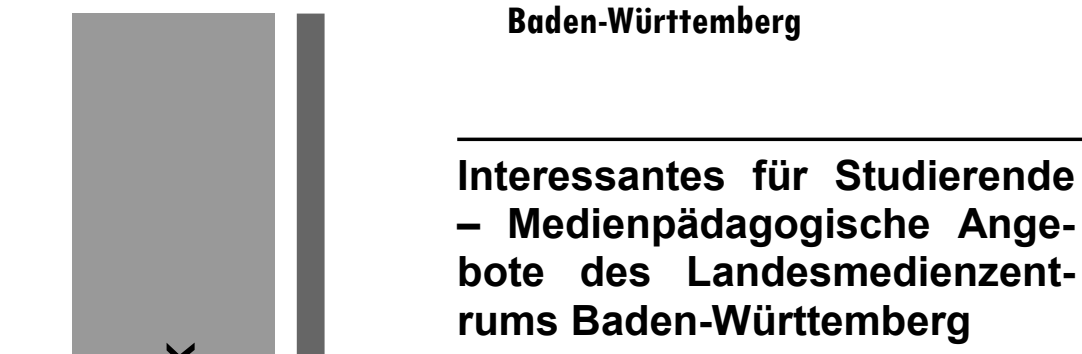

\section{CHRISTINE SATTLER}

Studierende von pädagogischen Fächern stehen spätestens im Berufsalltag vor neuen Herausforderungen. Um kompetente Ansprechpartner für Kinder und Jugendliche zu sein, müssen sie sich mit Medienpädagogik und den medialen Welten der jungen Generation auskennen. Sie sollen Schülerinnen und Schüler in Medienfragen anleiten und begleiten, Eltern Tipps für den Familienalltag geben und selbst in der Lage sein, digitale Medien souverän zu nutzen. Dabei ist es hilfreich, medienpädagogische Institutionen und deren Angebot zu kennen, um davon zu profitieren oder Informationen weitergeben zu können.

Eine dieser Institutionen in BadenWürttemberg ist das Landesmedienzentrum Baden-Württemberg (LMZ). Wir stellen Ihnen das LMZ und seine medienpädagogischen Angebote vor.

Das LMZ fördert landesweit mit dem Online-Portal www.mediaculture-online.de, mit Vorträgen, Workshops, pädagogischer Beratung und technischer Unterstützung die Medienkompetenz von Erwachsenen, Kindern und Jugendlichen. Als zentrales Kompetenzzentrum steht das LMZ Lehrkräften, Eltern, Schülerinnen und Schülern, Studierenden und Interessierten bei allen Fragen der Medienpädagogik und des pädagogischen Jugendmedienschutzes sowie der aktiven Medienarbeit zur Seite. Das LMZ orientiert sich am positiven Mediendiskurs und thematisiert vor allem die vielfältigen kreativen, kommunikativen, informativen und sozialen Möglichkeiten von digitalen Medien. Dabei kommt auch die Vermittlung von Werten nicht zu kurz, wie etwa die Ächtung jeglicher Gewalt und die Förderung eines wertschätzenden Umgangs miteinander auch im Internet oder über Handys und Smartphones.

Neben Angeboten zum pädagogischen Jugendmedienschutz und der praktischen Medienarbeit in Form von Informationsveranstaltungen, Workshops oder Fortbildungen hat das LMZ langfristige Peer-to-Peer-
Programme entwickelt - zum Beispiel das Schüler-Medienmentoren-Programm und das Programm „Medien! Eltern-Medienmentoren“. Der Peer-to-Peer-Ansatz hat sich als sehr erfolgreiche und nachhaltige Methode für Jugendliche, aber auch für Erwachsene erwiesen.

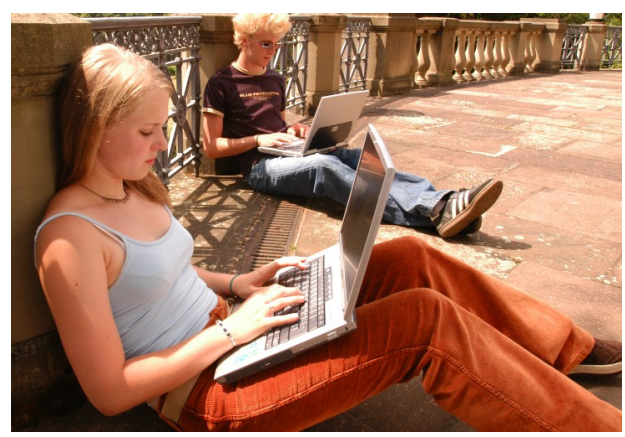

\section{Medienpädagogische Beratungs- stelle}

Zentrale Anlaufstation des LMZ ist die medienpädagogische Beratungsstelle. Lehrkräfte, Eltern und Pädagoginnen und Pädagogen außerhalb der Schule werden durch die persönliche Beratung bei der Medienerziehung unterstützt. Die Mitarbeiter der Beratungsstelle beantworten Fragen zu allen Aspekten jugendlicher Mediennutzung; zum Beispiel zu Sozialen Netzwerken wie Facebook, zu Online- oder Computerspielen, zu Cybermobbing, zu Datenschutz und Persönlichkeitsrechten, aber eben auch zu Methoden und Möglichkeiten der aktiven Medienarbeit mit Kamera, Mikrofon oder Blogs. Bei Bedarf und Interesse vermittelt das LMZ qualifizierte Referenten für Veranstaltungen wie Schülerworkshops oder Elternabende und unterstützt Schulen bei pädagogischen Tagen. Die Beratungsstelle empfiehlt aktuelle Informationen und Materialien für den Unterricht, den Familienalltag und die pädagogische Praxis und informiert über neueste Entwicklungen im Medienbereich.

Die medienpädagogische Beratungsstelle ist telefonisch von montags bis freitags von 9 bis 16 Uhr unter (0711) 2850-777 oder jederzeit per E-Mail unter beratungsstelle@lmzbw.de erreichbar. 


\section{Alles auf einen Klick - MediaCulture-Online für Studium und pädagogische Praxis}

Mit dem Online-Portal MediaCulture-online (www.mediaculture-online.de) bietet das LMZ eine einzigartige Plattform insbesondere für Studierende und Pädagogen. Das Portal informiert rund um die Themen Medienbildung, Medienpraxis und Medienkultur. Lehrerinnen und Lehrer, Studierende, Eltern und pädagogische Fachkräfte können sich über aktuelle Entwicklungen im Medienbereich informieren, erhalten Anregungen für die eigene Mediennutzung, praxiserprobte Anleitungen für den Unterricht oder den pädagogischen Alltag sowie zur eigenen Medienproduktion.

Studierende finden beispielsweise in der Online-Bibliothek digitale Literatur zu allen medienrelevanten Themen wie beispielsweise Medien und Politik, Medienpädagogik, Computer und Internet, Fernsehen und Video, Gewalt, Werbung und Journalismus. Im Tonarchiv finden sich Vorträge, politische Reden, Hörspiele, vertonte Gedichte, Radiobeiträge, Schülerproduktionen und vieles mehr im mp3Format.

Wissenspool Medien und Anleitungen zum Selbermachen

MediaCulture-Online ist $\mathrm{zu}$ einem Wissenspool herangewachsen, der fortlaufend aktualisierte Informationen zu den unterschiedlichsten Medien wie Computer und Internet, Handy, Film, Video, Radio oder Podcast sammelt. Neben Hintergrundinformationen finden sich dort auch praktische Anregungen, die das Selbermachen in den Mittelpunkt rücken, zum Beispiel Anleitungen, wie man eine Website gestaltet, einen Podcast, eine Radiosendung oder einen Trickfilm produziert. Darüber hinaus finden Interessierte auch didaktische Hinweise und Best-Practice-Beispiele etwa zum Einsatz von Whiteboards, Handys oder iPads für Unterricht, Schule und Jugendhaus.

Weitere Informationen zu den Angeboten des Landesmedienzentrums Baden-Württemberg finden Sie unter www.lmz-bw.de und unter www.mediaculture-online.de.

\section{Kontakt}

Landesmedienzentrum Baden-Württemberg

Medienpädagogische Beratungsstelle

Telefon: 0711 2850-777

E-Mail: beratungsstelle@1mz-bw.de 